FACTA UNIVERSITATIS

Series: Mechanical Engineering Vol. 15, No 3, 2017, pp. 397 - 411

https://doi.org/10.22190/FUME170505022R

Original scientific paper

\title{
CASTING IMPROVEMENT BASED ON METAHEURISTIC OPTIMIZATION AND NUMERICAL SIMULATION
}

UDC 621.7

\author{
Radomir Radiša ${ }^{1}$, Nedeljko Dučić ${ }^{2}$, Srećko Manasijević ${ }^{1}$, \\ Nemanja Marković ${ }^{3,4}$, Žarko Ćojbašić ${ }^{3}$ \\ ${ }^{1}$ Lola Institute Belgrade, Serbia \\ ${ }^{2}$ Faculty of Technical Sciences Čačak, University of Kragujevac, Serbia \\ ${ }^{3}$ Faculty of Mechanical Engineering, University of Niš, Serbia \\ ${ }^{4}$ Philip Morris Operations Serbia
}

\begin{abstract}
This paper presents the use of metaheuristic optimization techniques to support the improvement of casting process. Genetic algorithm (GA), Ant Colony Optimization (ACO), Simulated annealing (SA) and Particle Swarm Optimization (PSO) have been considered as optimization tools to define the geometry of the casting part's feeder. The proposed methodology has been demonstrated in the design of the feeder for casting Pelton turbine bucket. The results of the optimization are dimensional characteristics of the feeder, and the best result from all the implemented optimization processes has been adopted. Numerical simulation has been used to verify the validity of the presented design methodology and the feeding system optimization in the casting system of the Pelton turbine bucket.
\end{abstract}

Key words: Metaheuristic Optimization, Sand Casting, Feeders, Numerical Simulation

\section{INTRODUCTION}

The task of the optimization is to find the variables in which the target (criterion) function has extreme (minimum or maximum) value, with the limits, which define the space of potential solutions. Optimization is an integral part of natural processes. From the phenomena that take place at the level of micro-scale (e.g. crystallization, in which the molecules occupy the minimum energy position), to the evolutionary process leading to, through the principle of survival of the fittest, the individuals that are better adapted to the

Received May 05, 2017 / Accepted August 04, 2017

Corresponding author: Nedeljko Dučić

Faculty of Technical Sciences Čačak, University of Kragujevac, Svetog Save 65, 32000 Čačak

E-mail: nedeljko.ducic@ftn.kg.ac.rs 
conditions in the "environment" - all this serves as an inspiration for several metaheuristic optimization techniques. The implemented metaheuristic optimization methods are based on the idea that, by imitating nature, what should be looked for is the optimum complex function of several variables that represent the mathematical abstraction of a complex engineering problem. The idea developed in this paper is to apply metaheuristic optimization and advanced simulation for improvement of the casting process, which is tested on the problem of design and optimization of the feeder for sand casting of the Pelton turbine bucket.

When it comes to the implementation of metaheuristic optimization methods in the casting process, the spectrum of published research studies is very wide because of a large number of casting technologies and their respective complexity. Gravela et al. [1] presented ant colony optimization (ACO) for the solution of an industrial scheduling problem in an aluminum casting center. Santos et al. [2] presented the development of a computational algorithm (software) applied to maximizing the quality of steel billets produced by continuous casting. A mathematical model of solidification works integrated with a genetic search algorithm and a knowledge base of operational parameters. Surekha et al. [3] presented multi-objective optimization of green sand mould system using evolutionary algorithms, such as genetic algorithm (GA) and particle swarm optimization (PSO). Slavković et al. [4] presented application of learning machine methods in prediction and optimization of the wear rate of wear resistant casting parts. Dučić et al. [5] presented optimization of chemical composition in the manufacturing process of flotation balls based on intelligent soft sensing. Dučić et al. [6] presented optimization of the gating system for sand casting using genetic algorithm. The implementation of modern CAD/CAM software systems is frequent in the research projects of the casting process, as well as the combination of modern CAD/CAM software systems and methods of metaheuristic optimization. Dabade et al. [7] used MagmaSoft software for simulating the casting process and analyzing its various defects, by detecting the cause through simulation dimensionally and positionally different embodiments of the casting and the feeding systems. Jie et al. [8] used the Pro Cast software package to improve the casting process of aluminum alloy, and have concluded that the increase of molten metal temperature and of casting speed solves the problem of porosity. Nimbulkara and Dalu [9] presented the design of gating and feeding system with the objective of optimizing them by using the Auto-CAST X1 casting simulation software as well as of preparing the sand mold and casting the part, of comparing the simulated result and experimental results, of reducing the rejection rate and thus enabling the company to again start the production.

Unlike these studies, in this paper four metaheuristic optimization techniques have been considered, while the obtained results have been tested and verified with the numerical simulation of casting processes using the advanced MAGMA ${ }^{5}$ software.

\section{OPTIMIZATION ASPECTS OF FEEDING THE CASTING PART}

Optimal design of some system is a goal in more or less every engineering discipline. The imperative of optimal design of the feeding system of the cast is to reduce material consumption so that the feeder can successfully compensate shrinkage of the material in the mold cavity. Unlike the filling of the mold cavity, feeding is a long, slow process that 
is required during the contraction of the liquid that takes place on freezing. This process takes minutes or hours depending on the size of the casting. During freezing are present three different phases of the contraction volume, i.e. shrinkage: liquid contraction, solidification contraction and solid contraction (Fig. 1).

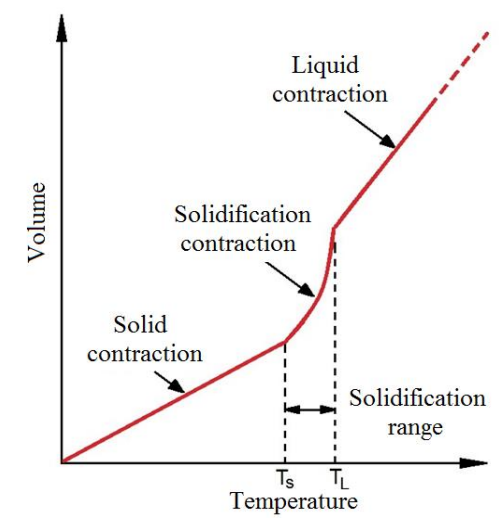

Fig. 1 Schematic illustration of three shrinkage regimes:

in the liquid; during freezing; and in the solid [10]

Volume contraction is manifested in side effects: internal cavities, surface deformation, surface craters. One of the indicators of casting process quality is continuity of molten metal flow in the area of solidification that is fed and compensating deficit caused by solidification. Failure in this process will result in deficiencies of the solidification process that is called porosity. In Fig. 2 just a generalized classification of porosity is given, as a result of metal shrinkage. Open defects, as a result of metal shrinkage, are result of cooling while metal is in liquid state and during solidification. These defects are large-volumed, so they are called macro-shrinkage. Closed shrinkage defects manifest themselves as an internal macroporosity and internal microporosity. Open defects are exclusively related to the process of metal shrinkage, while closed defects, in addition to process of metal shrinkage, are directly related to nucleation and growth of grains, as the characteristics of crystallization.

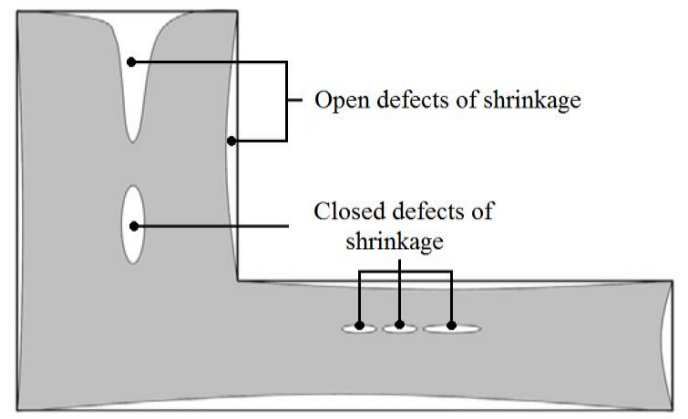

Fig. 2 Open and closed defects as a result of metal shrinkage 
The elimination of those side effects can be realized by the proper design of feeders which, after cooling, should be removed from the casting part. By exploring numerous literature references, as a general conclusion, the following sequence of activities in the cast feeding system design [11] is imposed:

(a) Representation of the casting as a collection of simple, plate-like shapes

- locate hot spots, and place a riser on each one

- for each plate-like shape, determine edges with and without end effect

(b) Determination of feeding zones, feeding paths and feeding dimensions.

(c) Determination of feeding distances

(d) Determination of riser sizes

Within this sequence are incorporated rules on valid feeding of casting part, which Cambell systematically exposes in his book [10]. Numerous literature sources are mainly based on two rules of feeding the cast:

(a) The feeder must solidify, at the earliest, at the same time as cast or, of course, later.

This rule is called Chvorinov's heat-transfer criterion.

(b) The feeder must contain sufficient molten metal to compensate to the casting part metal shrinkage, in the extent for which the aforementioned feeder is provided.

Metaheuristic optimization of a feeder is a certain synthesis of exposed rules and activities in the feeding system design, embedded in standard optimization subjects, such as the fitness function, and appropriate limits. As optimization techniques were used nature-inspired metaheuristic algorithms: Genetic algorithm (GA), Ant Colony Optimization (ACO), Simulated annealing (SA) and Particle Swarm Optimization (PSO).

\section{MetAHEURISTIC OPTIMIZATION TECHNIQUES}

Two major components of any metaheuristic algorithms are: intensification and diversification, or exploitation and exploration [12]. Diversification means to generate diverse solutions so as to explore the search space on a global scale, while intensification means to focus the search in a local region knowing that a current good solution is found in this region. A good balance between intensification and diversification should be found during the selection of the best solutions to improve the rate of algorithm convergence. The selection of the best ensures that solutions will converge to the optimum, while diversification via randomization allows the search to escape from local optima and, at the same time, increases the diversity of solutions. A good combination of these two major components will usually ensure that global optimality is achievable [13].

\subsection{Genetic algorithm (GA)}

Genetic algorithms (GA) [13] are probably the most popular and widely used metaheuristic optimization technique. They represent abstraction model of biological natural selection, based on Darwin's theory of evolution. Application of genetic algorithms assumes the use of concepts from nature such as crossover, mutation, recombination and selection in adaptive and artificial systems. Such genetic operators are important elements of the each problemsolving strategy by use of genetic algorithms. Genetic algorithms can be described by the following generic representation: 
Data: population size $\boldsymbol{N}$, crossover rate $\boldsymbol{\eta}_{\boldsymbol{c}}$ and mutation rate $\boldsymbol{\eta}_{\boldsymbol{m}}$.

Initialization: create initial population $\boldsymbol{P}=\left\{\boldsymbol{P}_{i}\right\}, \boldsymbol{i}=\mathbf{1} \ldots N$, and initialize the best solution Best $\leftarrow$ void.

WHILE \{stoppingcriterion not met

evaluate $\boldsymbol{P}$ and update the best solution Best.

initialize offspring population: $\boldsymbol{R} \leftarrow$ void.

create offsprings:

FOR $\boldsymbol{k}=1$ TO $\boldsymbol{N} / 2$ DO

selection stage: select parents $\boldsymbol{Q}_{\boldsymbol{1}}$ and $\boldsymbol{Q}_{2}$ from $\boldsymbol{P}$, based on fitness.

crossover stage: use crossover rate $\boldsymbol{\eta}_{c}$ and parents $\left(\boldsymbol{Q}_{1} ; \boldsymbol{Q}_{2}\right)$ to create offsprings $\left(S_{1} ; S_{2}\right)$.

mutation stage: use mutation rate $\boldsymbol{\eta}_{\boldsymbol{m}}$ to apply stochastic changes to $\boldsymbol{S}_{\boldsymbol{I}}$ and $S_{2}$ and create mutated offsprings $\boldsymbol{T}_{1}$ and $\boldsymbol{T}_{2}$.

Add $\boldsymbol{T}_{1}$ and $\boldsymbol{T}_{2}$ to offspring population: $\boldsymbol{R} \leftarrow \boldsymbol{R} U\left\{\boldsymbol{T}_{1}\right.$ and $\left.\boldsymbol{T}_{2}\right\}$.

Replace current population $\boldsymbol{P}$ with offspring population $\boldsymbol{R}: \boldsymbol{P} \leftarrow \boldsymbol{R}$.

Elitism: replace the poorest solution in $\boldsymbol{P}$ with the best solution in Best.

\subsection{Ant colony optimization (ACO)}

Social ants foraging behavior was the role model for development of ant colony optimization (ACO) technique [13]. Ants use chemical messenger called pheromone, being social insects that live together in organized colonies and that interact and communicate among themselves. While foraging, ants lay scent chemicals or pheromone and are able to follow the pheromone routes marked by other ants, indicating the trail to food source. The ants follow the route with higher pheromone concentration, and as more and more ants follow the same route, it becomes the favored path with enhanced pheromone which is likely the shortest or more efficient path. Evolving, the system converges to a self-organized state. Generic representation of ant colony algorithm is:

Data: Population size $N$, set of components $C=\left\{C_{l}, \ldots, C \boldsymbol{n}\right\}$, evaporation rate evap. Initialization: amount of pheromones for each component $\boldsymbol{P H}=\left\{\boldsymbol{P} \boldsymbol{H}_{1}, \ldots, \boldsymbol{P \boldsymbol { H } _ { n }}\right\}$; best solution Best.

WHILE \{stopping criterion not met

initialize current population, $\boldsymbol{P}=$ void .

Create current population of virtual solutions $\boldsymbol{P}$ :

FOR $i=1$ TO $N$ DO

Create feasible solution $S$.

Update the best solution, Best $\leftarrow$ void.

Add solution $\boldsymbol{S}$ to $\boldsymbol{P}: \boldsymbol{P} \leftarrow \boldsymbol{P} U \boldsymbol{S}$

Apply evaporation:

FOR $\boldsymbol{j}=1$ TO $\boldsymbol{n}$ DO

$$
\mathrm{PH}_{\mathrm{j}}=\mathrm{PH}_{\mathrm{j}} \cdot(1-\text { evap })
$$

Update pheromones for each component:

FOR $i=1$ TO $N$ DO

FOR $\boldsymbol{j}=1$ TO $\boldsymbol{n}$ DO

if component $\mathrm{C}_{\mathrm{i}}$ is part of solution $\mathrm{P}_{\mathrm{j}}$, then update pheromones for this component: $\mathrm{PH}_{\mathrm{j}}=\mathrm{PH}_{\mathrm{j}}+$ Fitness $\left(\mathrm{P}_{\mathrm{j}}\right)$ 


\subsection{Simulated annealing (SA)}

Simulated annealing (SA) optimization was designed using analogy with metal annealing [14], and is a technique possessing main ability to avoid being trapped in local optima unlike deterministic optimization techniques. It is an optimization method which is alike the process of warming up a solid to melting, then followed by cooling it down until it crystallizes into a perfect lattice. Simulated annealing could be considered as Markov chain following search [12], which converges under appropriate settings. With each search move, moving trace a piecewise path, acceptance probability is assessed, accepting alterations improving the objective function and also keeping some changes that do not improve the objective [13]. Generic representation of simulated annealing is described by:

Data: initial approximation $\boldsymbol{X}_{\boldsymbol{0}}$, initial temperature $\boldsymbol{T}$, number of iteration for a given temperature $\boldsymbol{n} \boldsymbol{T}$.

Optimal solution: $\boldsymbol{X}_{\text {best }} \leftarrow \boldsymbol{X}_{\boldsymbol{0}}$.

WHILE $\{$ stopping criterion not met

$\boldsymbol{n}=0 ; \boldsymbol{i}=0$;

WHILE $(\boldsymbol{n}<\boldsymbol{n} \boldsymbol{T})$ DO

Choose a new approximation $\boldsymbol{Y}$

Accept or reject the new approximation based on the Metropolis rule: $\boldsymbol{X}_{\boldsymbol{i}+\boldsymbol{I}}$ $=\mathrm{G}\left(\boldsymbol{X}_{\boldsymbol{i}}, \boldsymbol{Y}, \boldsymbol{T}\right)$

update optimal solution: if $\boldsymbol{X}_{i+1}$ is better than $\boldsymbol{X}_{\text {best }}$, then $\boldsymbol{X}_{\text {best }} \leftarrow \boldsymbol{X}_{i+1}$

next n-iteration $(\boldsymbol{n} \leftarrow \boldsymbol{n}+\boldsymbol{1})$

update temperature $\boldsymbol{T}$

next $\boldsymbol{T}$-iteration $(\boldsymbol{i} \leftarrow \boldsymbol{i}+\boldsymbol{1})$

\subsection{Particle swarm optimization (PSO)}

Particle swarm optimization (PSO) is a technique developed on resembling swarm behavior, such as fish and bird schooling [13]. PSO has generated a lot of attention in the field of swarm intelligence. System is initialized with a population of random solutions and searches for optima by updating generations. The potential solutions, called particles, fly through the problem space by following the current optimum particles. Each particle keeps track of its coordinates which are associated with the best solution (fitness), and its value is also stored. Best fitness value is taken as final solution. Numerous PSO variants exist, as well as hybrid algorithms combining PSO with other algorithms. PSO is described by the following representation:

Data: population size $\boldsymbol{N}$, personal-best weight $\boldsymbol{\alpha}$, local-best weight $\boldsymbol{\beta}$, global-best weight $\gamma$, correction factor $\boldsymbol{\varepsilon}$.

Initialization: create initial population $\boldsymbol{P}$.

WHILE \{stopping criterion not met\}

Select the best solution from the current P: Best.

Select global-best solution for all particles: $\mathrm{B}^{\mathrm{G}}$.

Apply swarming:

\section{FOR $i=1$ TO $\boldsymbol{N}$ DO}

Select personal-best solution for particle $\boldsymbol{X}_{i}: \boldsymbol{B}_{i}{ }^{\boldsymbol{P}}$.

Select local-best solution for particle $\boldsymbol{X}_{i}: \boldsymbol{B}_{i}{ }^{\boldsymbol{L}}$. 
Compute velocity for particle $\boldsymbol{X}_{\boldsymbol{i}}$ :

FOR $j=1$ TO DIM DO

Generate correction coefficients: $\boldsymbol{a}=\boldsymbol{\alpha} \cdot \operatorname{rand}() ; \boldsymbol{b}=\boldsymbol{\beta} \cdot \operatorname{rand}()$; $c=\gamma \cdot \operatorname{rand}()$.

Update velocity of particle $\boldsymbol{X}_{i}$ along dimension $\boldsymbol{j}$ :

$V_{i j}=V_{i j}+a \cdot\left(B_{i j}{ }^{P}-x_{i j}\right)+b \cdot\left(B_{i j}{ }^{L}-x_{i j}\right)+c \cdot\left(B_{j}{ }^{G}-x_{i j}\right)$

Update position of particle $\boldsymbol{X}_{i}: \boldsymbol{X}_{\boldsymbol{i}}=\boldsymbol{X}_{\boldsymbol{i}}+\boldsymbol{\varepsilon} \cdot \boldsymbol{V}_{\boldsymbol{i}}$

\section{OPTIMIZATION PROCESSES}

The proposed methodology of combining metaheuristic optimization and advanced casting simulation is applied to the problem of optimization of geometry of a feeder for casting Pelton turbine bucket (its CAD model is shown in Fig. 3). Optimization is based on the precise formulation of the objective function and constraints, and abovementioned techniques of metaheuristic optimization were implemented.

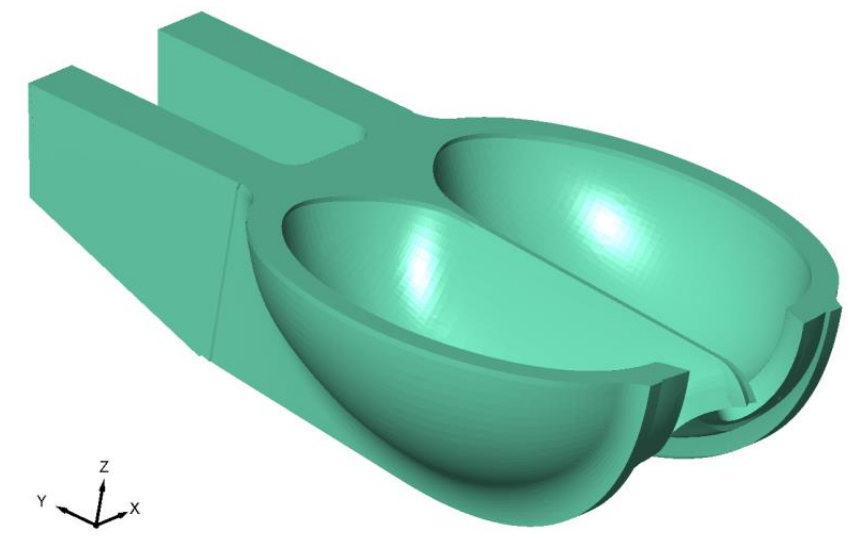

Fig. 3 CAD model of buckets Pelton turbine

\subsection{Formulation of the optimization problem}

The formulation of the optimization problem is actually defining the fitness function. Defining the fitness function is one of the major steps of the optimization process. Creating the fitness function is based on the rule: The heat-transfer requirement. The heat-transfer requirement for successful feeding can be stated as follows: the freezing time of the feeder must be at least as long as the freezing time of the casting [10]. Casting solidification time can be predicted using Chvorinov's Rule shown by the equation [11]:

$$
t_{s}=C\left(\frac{V}{A}\right)^{2},
$$

where $t_{s}$ is the total solidification time of the part or feeder, $C$ is a mold constant, $V$ is the volume of metal, and $A$ is the total surface area of the part or feeder. As the feeder serves 
to compensate for the volume shrinkage in the casting part, it needs to be the final link of the solidification chain, i.e. it should be the last to solidify in directional solidification process. The longest solidification time for a given volume is the one where the shape of the part has the minimum surface area. Feeder module $\left(M_{\text {feeder }}\right)$ is taken to be for a minimum of $20 \%$ higher than the module of casting part, i.e. module of part which feeds [10]:

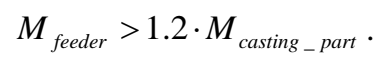

The modulus of a designed feeder is given by Eq. (3), based on the dimensional characteristics (Fig. 4):

$$
M_{\text {feeder }}=\frac{V_{\text {feeder }}}{P_{\text {feeder }}}=\frac{H \cdot\left(R^{2}+R \cdot r+r^{2}\right)+2 \cdot R \cdot l \cdot H-(R-r) \cdot H \cdot l}{3 \cdot\left(R^{2}+r^{2}+\sqrt{H^{2}+(R-r)^{2}} \cdot(R+r)\right)+2 \cdot l \cdot \sqrt{H^{2}+\left(\frac{R-r}{2}\right)^{2}}}
$$
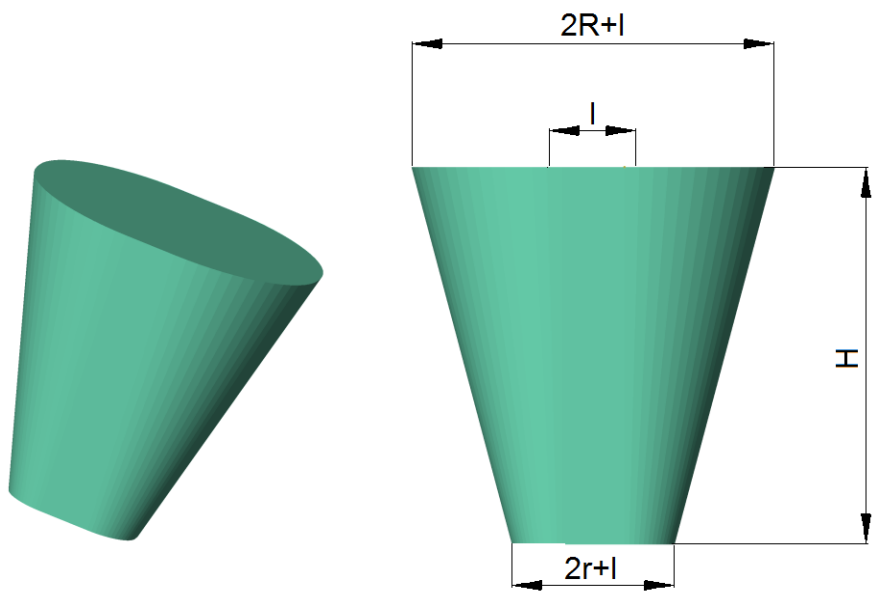

Fig. 4 CAD model of feeder and dimensional characteristics

Based on the values of volume and surface of the casting part, obtained by analyzing the CAD model $\left(V_{c}=4269327.33 \mathrm{~mm}^{3}\right.$ and $\left.A_{c}=348116.32 \mathrm{~mm}^{2}\right)$, the module of the casting part is $M_{\text {casting_part }}=12.26 \mathrm{~mm}$. Eq. (4) gives the final expression of fitness function $F(R, r, H, l)$, where all values are entered in $[\mathrm{mm}]$, that minimizes:

$$
F=\frac{H \cdot\left(R^{2}+R \cdot r+r^{2}\right)+2 \cdot R \cdot l \cdot H-(R-r) \cdot H \cdot l}{3 \cdot\left(R^{2}+r^{2}+\sqrt{H^{2}+(R-r)^{2}} \cdot(R+r)\right)+2 \cdot l \cdot \sqrt{H^{2}+\left(\frac{R-r}{2}\right)^{2}}}-12.26>0
$$




\subsection{Constraints of optimizations parameter}

First constraint is based on the rule Mass-transfer (volume) requirement. The fulfillment of the conditions given in rule "The heat-transfer requirement", does not guarantee the fulfillment of the conditions given in rule "Mass-transfer (volume) requirement". Although the feeder may have been provided of such a size that it theoretically would contain liquid until after the casting is solid, in fact it may still be too small to deliver the volume of feed liquid that the casting demands. Thus, it will be prematurely sucked dry, and the resulting shrinkage cavity will extend into the casting. This is because they are themselves freezing at the same time as the casting, depleting the liquid reserves of the reservoir. Effectively, the feeder has to feed both itself and the casting. We can allow for this in the following way. If we denote efficiency $\varepsilon$ of the feeder as the ratio (volume of available feed metal) / (volume of feeder, $V_{f}$ ) then the volume of feed metal is, of course $\varepsilon \cdot V_{f}$. If the solidification shrinkage of the liquid is $\alpha$, during freezing, then the feed demand from both the feeder and casting together is $\alpha\left(V_{f}, V_{c}\right)$, and hence [10]:

$$
\varepsilon V_{f}=\alpha\left(V_{f}+V_{c}\right) .
$$

Solving for the feeder volume yields:

$$
V_{f} \geq \frac{\alpha V_{c}}{\varepsilon-\alpha} \geq 0.4 V_{c} \Rightarrow H \cdot\left(R^{2}+R \cdot r+r^{2}\right)+2 \cdot R \cdot l \cdot H-(R-r) \cdot H \cdot l \geq 0.25 V_{c},
$$

where $\alpha$ is the solidification shrinkage of the liquid during freezing ( $\alpha=4$, for steels the fcc structure applies above $0.1 \%$ carbon where the melt solidifies to austenite) and $\varepsilon=20 \%$ for appropriate feeder. Relation (6) is a constraint 1 in the optimization process of geometry feeders.

In addition to the abovementioned constraints, other constraints are referred to the mutual relations of values which are optimized and available space on the casting part.

Table 1 The other constraints

\begin{tabular}{lc}
\hline Constraint 2 & $2 r+l<50$ \\
Constraint 3 & $H<155$ \\
Constraint 4 & $2 R+l<160$ \\
\hline
\end{tabular}

\subsection{Results of the optimization process}

The results of the carried out optimization processes are relatively similar and their overview is given in Table 2. Simultaneous application of several metaheuristic optimization techniques allows for efficient verification of obtained optimization results.

Table 2 Results of optimization process

\begin{tabular}{ccccc}
\hline Optimization value & GA & ACO & SA & PSO \\
\hline $\boldsymbol{H}$ & 153 & 148 & 154 & 150 \\
$\boldsymbol{R}$ & 61 & 58 & 55 & 57 \\
$\boldsymbol{r}$ & 20 & 22 & 23 & 19 \\
$\boldsymbol{l}$ & 26 & 24 & 28 & 27 \\
\hline
\end{tabular}


Based on the obtained results of taken optimizations, the following values of optimized sizes have been adopted: $H=155 \mathrm{~mm}, R=60 \mathrm{~mm}, r=20 \mathrm{~mm}$ and $l=25 \mathrm{~mm}$. According to these values, CAD model of feeder was created and embedded into assembly of the entire gating system with a casting part.

\section{NUMERICAL SIMULATION AS A VERIFICATION OF THE OPTIMIZATION RESUlT}

Instead of experimental trial-and-error, the results of mold cavity filling, solidification, critical spots, formation of residual stresses, etc. now optimized in a virtual computer environment with various conditions. Using numerical simulations in the development of casting technology not only shortens the time necessary for product development but also reduces expenditures, reduces risks from defects, etc. Modern digital tools enable a relatively fast attainment of optimized solutions for casting technology, which results in stable manufacture with lower expenditures [15-19]. Digital tools using for optimization of the technical parameters influence the macro and microstructure of a cast. Digital tools allow different technological parameters of a casting process to be tested. Hence, to perform the simulation, it is necessary to provide a 3D geometric model of the casting and other components (tools for casting, gating system, feeders, etc.) and technological parameters (casting temperature, casting time, alloy composition, etc.). Based on the results of experimental investigations, the optimization of relevant technological parameters and their implementation under industrial conditions showed that the properties of a casting, in practice, could be significantly improved. The results show that, under industrial conditions, digital tools provide satisfactory solutions in a short period, starting virtually from scratch. It was shown that the employment of these software packages brings great advantages compared to the conventional manner of adopting new products. For instance, the results obtained in a rapid generation of model elements significantly accelerated the work and improved the productivity of engineers, reduced the time for adopting new products, reduced the scope of traditional prototype testing (creating prototypes and their testing), which is expensive and time consuming, causing standstills in manufacture, and reduced expenditures for adopting new products. The digital tool (in our case used MAGMA ${ }^{5}$ ) package itself automatically generates a mesh. The software user can set the fineness of the mesh, i.e., the number of mesh elements by defining the minimum size of the desired elements in all three directions of the coordinate system (Fig. 5).

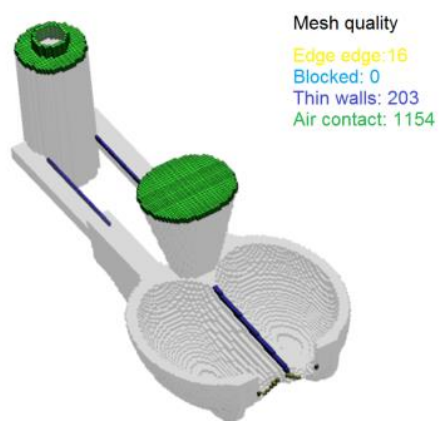

Fig. 5 Mesh Quality 
The finer the mesh, i.e., the more elements it has, the more accurate the calculation of the simulation is, but the simulation time is longer. The prepared mesh is used for further calculation. For each element, i.e., part of the mesh, differential equations used to calculate its physical and thermal parameters and the results obtained are boundary conditions for the calculation of the parameters in the neighboring element. Thus, the computer calculates element by element in $3 \mathrm{D}$ coordinates and finally integrates all the partial results for the overall geometry. The MAGMA ${ }^{5}$ software has many criteria for casting process simulation, part filling, but the results of two criteria are present in this paper:

- Fill_Temp, whose results show the distribution of temperatures in the mold cavity from the start to the end of filling; this provides the possibility of monitoring the temperature distribution throughout the mold cavity at any time (Fig. 6).

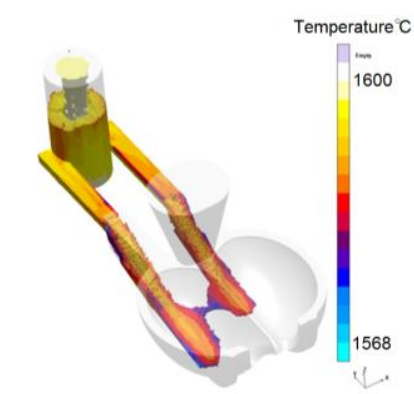

a)

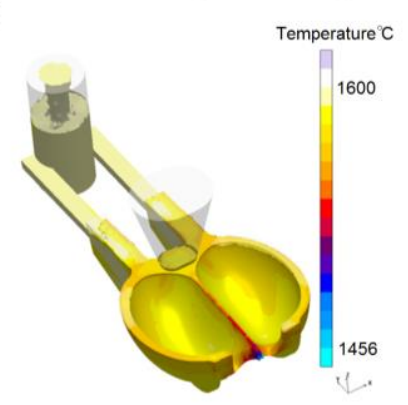

c) b)
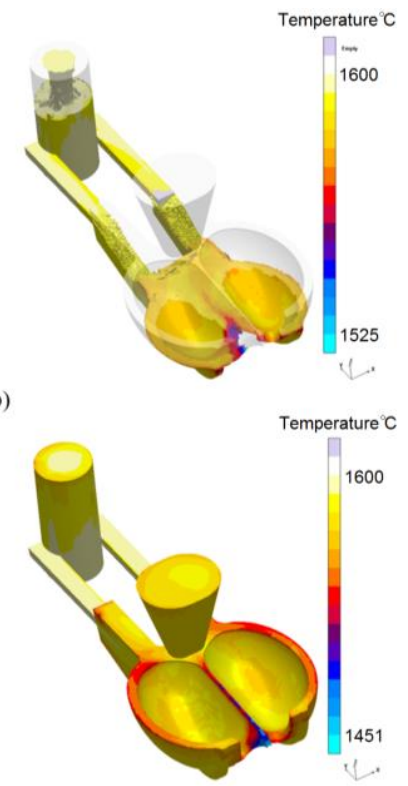

d)

Fig. 6 Fill_Temp - filling: a) $25 \%$, b) $50 \%$, c) $75 \%$, and d) $100 \%$

- Fill_Tracer, which shows which portion of the metal in the casting passed through ingate. The distribution of the melt in the simulation is visible because the respective melt fractions from each ingate are highlighted in different colors. This means that possible changes of the gate positions and gate cross sections as well as changes in the angles at which different sections of the gates are connected could be simulated and the resulting impact on the filling of the mold could be made easily visible, thus substantially facilitating an optimized gating design (Fig. 7). 


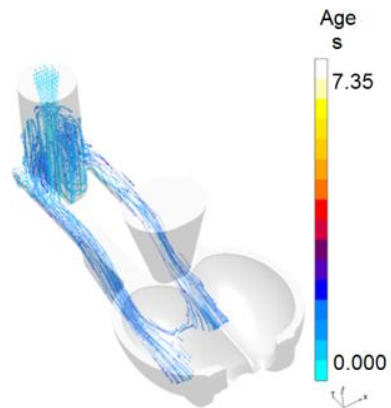

a)

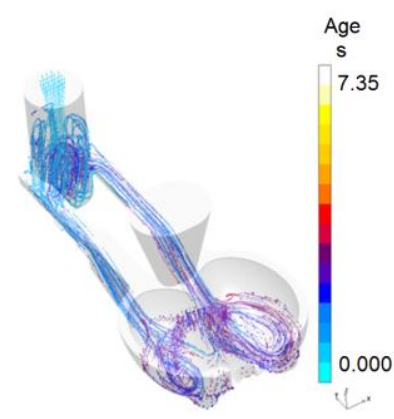

c)

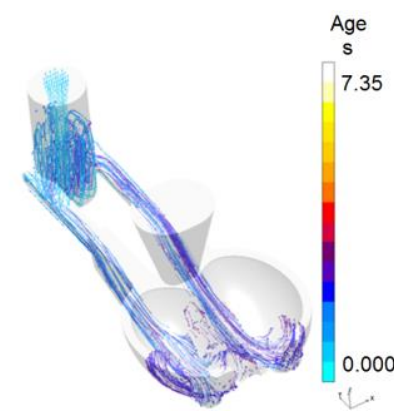

b)

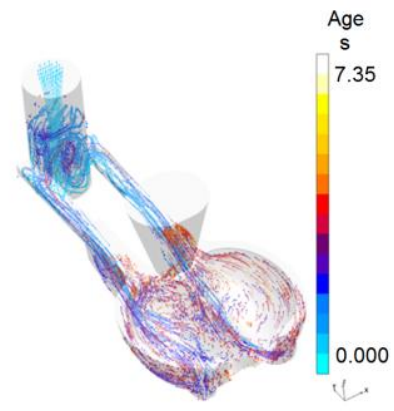

d)

Fig. 7 Fill_Tracer - filling: a) $25 \%$, b) $50 \%$, c) $75 \%$ and d) $100 \%$

In order to define the solidification, i.e., the cooling process of bucket casting, necessary parameters and boundary conditions are entered (the time or temperature at which the solidification simulation stops specified). Generally, it is possible to perform simulation without the results of casting solidification. However, if casting simulation is performed before solidification simulation, then the results and temperatures reached at the end of mold filling are taken as the basis for solidification simulation (the Fill_Temp file) [15]. In the used MAGMA ${ }^{5}$ version presented in this paper, this Fill_Temp file exists in the system, which considerably simplifies the procedure. The solidification results are also shown through several criteria: Solid_Temp (shows temperature distribution within the selected material in the casting system during solidification), Hotspot (determines isolated regions of residual melt at any time during solidification), and Porosity (can visualize porosities in the casting). Using the Solidification Criteria Results after the simulation is terminated, the program automatically calculates several criteria that can be selected at each point. Displaying the criteria results helps find defects in the casting and analyze the solidification behavior. The solidification time is $50 \mathrm{~min}$. This paper presents the results of the following criteria: Solid_Temp (Fig. 8), Hotspot and Porosity. 


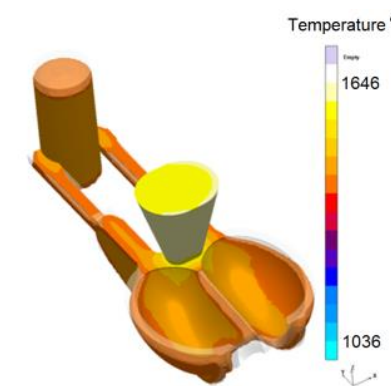

a)

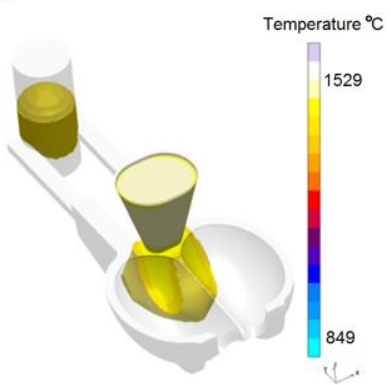

c)

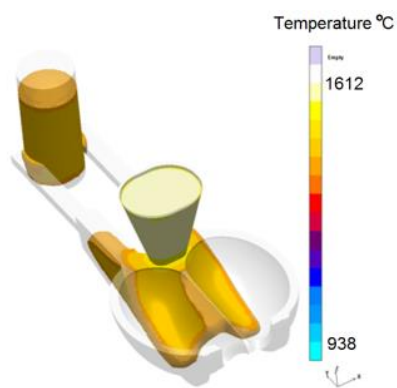

b)

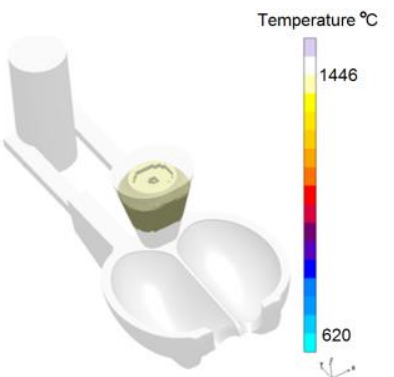

d)

Fig. 8 Solid_Temp - solidification, a) $25 \%$, b) $50 \%$, c) $75 \%$ and d) $100 \%$

The results show that the solidification process develops in a directed way and that the last parts that solidify are pour cup and feeder. Using the Hotspot criterion, isolated regions of residual melt (Fig. 9a) are determined at any time during solidification. If the feeding calculation option in the simulation setup is checked, this criterion helps to detect porosities in these residual melt regions. The unit is solidification time in seconds (s, color scale). This can determine the time during solidification at which a particular hot spot develops. It can be clearly seen in Fig. $9 \mathrm{~b}$ that the solidification process develops according to expectations and that the last points of solidification are in feeders. In this case, the solidification time is $47 \mathrm{~min}$.

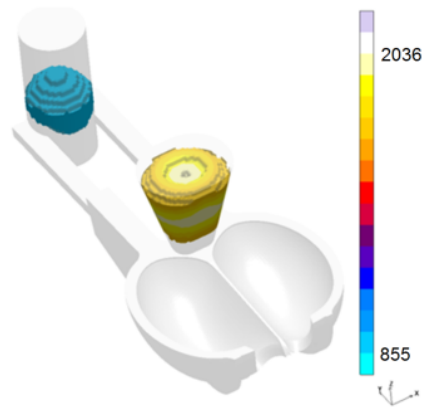

a)

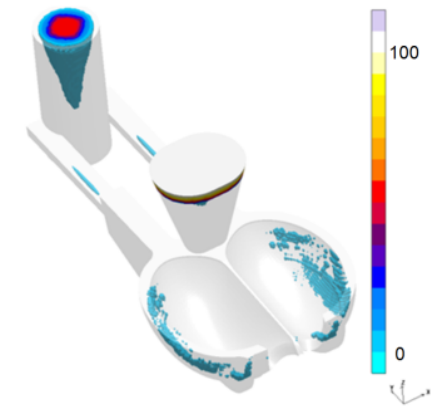

b)

Fig. 9 Solidification results, a) Hotspot and b) Porosity 
Using the Porosity criterion, porosities in the casting can be visualized (Fig. 9b). The portion of porosities at the end of solidification simulation is displayed. The unit is percent $(\%$, color scale). The unfilled and thus porous regions are displayed in white. An analysis of porosity problems is the most reliable when using this criterion.

\section{CONCLUSION}

This paper presents a methodology for improvement of casting process, demonstrated on the problem of design and optimization of the feeder's geometry in the process of casting Pelton turbine bucket. Four methods of metaheuristic optimization were used: Genetic algorithm (GA), Ant Colony Optimization (ACO), Simulated annealing (SA) and Particle Swarm Optimization (PSO), which gave satisfactory and very similar results, mutually confirming validity of the obtained solution. Based on the obtained results geometry of feeder was dimensioned and CAD model of feeder was created. Performed numerical simulations confirm the correctness of the designed optimized feeding system according to all criteria.

Acknowledgement: The paper is a part of the research done within the projects TR35037, TR35015, TR35016 and TR35005.

\section{REFERENCES}

1. Gravela, M., Priceb, L., W., Gagné, C., 2002, Scheduling continuous casting of aluminum using a multiple objective ant colony optimization metaheuristic, European Journal of Operational Research, 143 (1), pp. 218-229.

2. Santosa, C.A., Spimb, J.A., Garcia, A., 2003, Mathematical modeling and optimization strategies (genetic algorithm and knowledge base) applied to the continuous casting of steel, Engineering Applications of Artificial Intelligence, 16 (5-6), pp. 511-527.

3. Surekha, B., Kaushik, K.L., Panduy, K.A., Vundavilli, R.P., Parappagoudar, B. M., 2012, Multiobjective optimization of green sand mould system using evolutionary algorithms, The International Journal of Advanced Manufacturing Technology, 58 (1), pp. 9-17.

4. Slavković, R., Jugović, Z., Dragićević, S., Jovičić, A., Slavković, V., 2013, An application of learning machine methods in prediction of wear rate of wear resistant casting parts, Computers and Industrial Engineering, 64(3), pp. 850-857.

5. Dučić, N., Ćojbašić, Ž., Slavković, R., Jordović, B., Purenović, J., 2016, Optimization of chemical composition in the manufacturing process of flotation balls based on intelligent soft sensing, Hemijska industrija, 70(6), pp. 603-614.

6. Dučić, N., Ćojbašić, Ž., Manasijević, Radiša, R., Slavković, R., Milićević, I., 2017, Optimization of the Gating System for Sand Casting Using Genetic Algorithm, International journal of metalcasting, 11 (2), pp. 222-265.

7. Dabade, A. U., Bhedasgaonkar, C.R., 2013, Casting Defect Analysis using Design of Experiments (DoE) and Computer Aided Casting Simulation Technique, Procedia CIRP, 7, pp. 616-621.

8. Jie, Z., Dongqi, Z., Pengwei, W., Gang, W., Feng, L., Penglong, D., 2014, Numerical Simulation Research of Investment Casting for TiB2/A356 Aluminum Base Composite, Rare Metal Materials and Engineering, 43 (1), pp. 47-51.

9. Nimbulkara, L.S., Dalu, S.R., 2016, Design optimization of gating and feeding system through simulation technique for sand casting of wear plate, Perspectives in Science, 8, pp. 39-42.

10. Campbell, J., 2011, Complete casting Handbook, 1st Edition, Elsevier, 661 p.

11. Steel Founders' Society of America, 2001, Feeding \& Risering Guidelines for Steel Castings, Steel Founders' Society of America, $11 \mathrm{p}$. 
12. Blum, C., Roli, A., 2003, Metaheuristics in combinatorial optimization: Overview and conceptual comparison, ACM Comput. Surv., 35, pp. 268-308.

13. Yang, X, -S., 2011, Metaheuristic Optimization, Scholarpedia, 6(8), 11472.

14. Kirkpatrick, S., Gelatt, C.D., Vecchi, M.P., 1983, Optimization by simulated annealing, Science, 220(4598), pp. 671-680.

15. Official website of MagmaSoft software for casting simulation: http://www.magmasoft.com/en/ (Last access $28^{\text {th }}$ April 2017)

16. Manasijević, S., Radiša, R., Petrović, B., 2008, Skil and intuition of foundrymen or computer assisted casting, Livarstvo-Foundry, 47(1), pp. 1-11.

17. Radiša, R., Marković, S., Pristavec, J., Kvrgić, V., Manasijević, S., 2008, Use of CAE Techniques in Virtual design of Metalcasting technology-saving in Serbian Foundries, Livarstvo-Foundry, 47(1), pp. $12-24$.

18. Radiša, R., Manasijević, S., Marković, S., 2009, Optimization technology of metal casting using software tool, Livarstvo-Foundy, 48(3), pp. 30-36.

19. Radiša, R., Gulišija Z., Manasijević, S., 2009, Optimization of casting process, Machine Design, 1, pp. $111-114$. 